SUPPORTING ONLINE MATERIAL

\title{
Unusual Hydrogen Bonding in Water-filled Carbon Nanotubes
}

\author{
Oleg Byl, ${ }^{1}$ Jin-Chen Liu, ${ }^{1}$ Yang Wang, ${ }^{2}$ Wai-Leung Yim, ${ }^{3}$ J. Karl Johnson, ${ }^{2,4}$ \\ and John T. Yates, Jr. ${ }^{1 *}$ \\ ${ }^{1}$ Department of Chemistry, \\ Surface Science Center, \\ University of Pittsburgh, \\ Pittsburgh, PA 15260 \\ ${ }^{2}$ Department of Chemical Engineering, \\ University of Pittsburgh, \\ Pittsburgh, PA 15261 \\ ${ }^{3}$ Carl von Ossietzky Universität Oldenburg \\ Institut für Reine und Angewandte Chemie \\ Carl-von-Ossietzky Str. 9-11 \\ 26129 Oldenburg, Germany \\ ${ }^{4}$ National Energy Technology Laboratory, \\ Pittsburgh, PA 15236
}




\section{Computational Methods}

\section{Potential models}

(1) Molecular models for water

We used the rigid SPC/E ${ }^{1}$ and flexible SPC/E ${ }^{2}$ water models for $\mathrm{MC}$ and $\mathrm{MD}$ simulations, respectively. The flexibility was introduced by the intra-molecular potential proposed by Toukan and Rahman ${ }^{3}$. The Hamiltonian we used for the flexible SPC/E water model was adapted from Praprotnik et al. ${ }^{2}$ and is given by

$$
\begin{aligned}
H=\sum_{i} & \frac{p_{i}^{2}}{2 m_{i}}+\sum_{k} \sum_{l=1}^{2} D_{e}\left[1-\exp \left(-\alpha \Delta r_{l_{k}}\right)\right]^{2}+\frac{1}{2} \sum_{k} k_{\theta} \Delta r_{3_{k}}{ }^{2} \\
& +\sum_{k} k_{r \theta} \Delta r_{3_{k}}\left(\Delta r_{1_{k}}+\Delta r_{2_{k}}\right)+\sum_{k} k_{r r} \Delta r_{1_{k}} \Delta r_{2_{k}} \\
& +\sum_{i>j}\left\{\frac{q_{i} q_{j}}{4 \pi e_{0} r_{i j}}+4 \varepsilon_{i j}\left[\left(\frac{\sigma_{i j}}{r_{i j}}\right)^{12}-\left(\frac{\sigma_{i j}}{r_{i j}}\right)^{6}\right]\right\} .
\end{aligned}
$$

The first summation is over all atoms, $m_{i}$ and $p_{i}$ are the mass and momentum of the $i$ th atom, respectively. The indices $k$ and $l$ run over all molecules, and all sites on a molecule, respectively. $\Delta r_{1_{k}}$ and $\Delta r_{2_{k}}$ are the changes in the $\mathrm{O}-\mathrm{H}$ bond lengths relative to the equilibrium bond length, $\Delta r_{3_{k}}$ is the stretch in the $\mathrm{H}-\mathrm{H}$ distance of the $k$ th molecule, $D_{e}$ is the depth of the Morse potential, $\alpha=\left(\sqrt{k_{r}} / 2 D_{e}\right), k_{r}, k_{\theta}, k_{r \theta}$ and $k_{r r}$ are the intramolecular force constants, $q_{i}$ is the charge on the $i$ th atom, $r_{i j}$ is the distance between the $i$ th and $j$ th atoms on different molecules, and $\varepsilon_{i j}$ and $\sigma_{i j}$ are the the Lennard-Jones potential parameters. Values for these parameters can be found in reference ${ }^{2}$. We note that there is an error in the sign of the Morse potential given in reference ${ }^{2}$.

The fluid-fluid interaction potentials were truncated at $1.42 \mathrm{~nm}\left(4.5 \sigma_{f f}\right)$. We have not included long-range electrostatic corrections; a simulation in a much longer nanotube with a fluid-fluid cutoff of $3.0 \mathrm{~nm}$ gave essentially identical results. We ran several test calculations using the reaction field method for accounting for electrostatic long-range interactions, but these simulations, with a range of different dielectric constants, gave results that were very similar to simulations with no long-range corrections. We have also compared our calculated IR spectra for bulk water with published simulations that utilized the Ewald technique for long-range electrostatics. Our results are described below.

(2) Carbon nanotube model

We used a structureless carbon nanotube in our simulations. The length of the nanotube was set to $3.06 \mathrm{~nm}$ in the $\mathrm{z}$ direction and periodic boundary conditions were applied in that direction. The radii for the $(8,8)$ and $(10,10)$ nanotubes are 0.542 and $0.678 \mathrm{~nm}$, respectively. Water-nanotube interactions were modeled by an integrated 
potential ${ }^{4}$. Both oxygen and hydrogen atoms interact with the tube. The parameters given by Martí and Gordillo ${ }^{5}$ were used in our simulations.

\section{Simulation details}

(1) Monte Carlo simulations

For simulation of water confined in a nanotube, we first used the GCMC simulation technique ${ }^{6}$ to get the adsorption isotherms in the nanotubes at a temperature of $298 \mathrm{~K}$. The isotherms are shown in Fig. S1. For the length of the nanotube we used, we obtained 44,55 and 77 molecules for the $(8,8)$ and $(10,10)$ SWNTs at pressure close to saturation, respectively. For the simulation of bulk amorphous ice, a cubic box with the box length of $2.917 \mathrm{~nm}$ containing 780 molecules was used. Then we performed parallel tempering Monte Carlo simulations ${ }^{7}$ in the NVT ensemble over the temperature range from $123 \mathrm{~K}$ to $298 \mathrm{~K}(318 \mathrm{~K}$ for $(10,10)$ nanotube $)$. Parallel tempering was required to equilibrate the system at low temperatures because of the existence of very many deep local minima in the potential energy landscape. We used an orientational bias technique ${ }^{7}$ for both GCMC and parallel tempering MC simulations to improve the sampling of the molecular orientations. Total run length of $1 \times 10^{8}$ configurations were used for GCMC simulations with half of which for equilibration. For the parallel tempering Monte Carlo simulations, we used run length of $4 \times 10^{8}$ configurations, half of which were used to relax the system. A configuration consisted of an attempt to make either a translation or rotation of a molecule for NVT simulation and additional creation and deletion for GCMC simulation. These moves were chosen with equal probability. The replica swap between adjacent replicas was made every 1000 (2000) configurations for confined (bulk) water simulations.

Additional GCMC simulations were carried out to model the adsorption of water in a $(10,10)$ SWNT at $183 \mathrm{~K}$. Results of these calculations are shown in Fig. S2. The simulations required much longer times to equilibrate than the $298 \mathrm{~K}$ isotherms shown in Fig. S1. Note that the low temperature induces large hysteresis in the adsorption and desorption branches. This is symptomatic of inefficient exploration of phase space and could be addressed with more advanced simulation techniques, such as hyper-parallel tempering Monte Carlo, but the results shown in Fig. S2 are sufficiently accurate to verify that a stacked ring structure of water molecules will fill nanotubes at the temperatures probed in the experiments. Filling of the nanotube takes place through an apparent first-order transition from an empty nanotube to a full nanotube. The water in the $(10,10)$ nanotube can exist as 7 -membered rings (heptagons), 8 -membered rings (octagons) or as octagons plus an axial phase, depending on the pressure.

We present additional structural information for water in various nanotubes in Figs. S3 and S4. Water in $(8,8),(9,9)$, and $(11,11)$ nanotubes has been studied and the bond angles and bond lengths are plotted. The results are qualitatively similar to those found for water in the $(10,10)$ SWNT. 


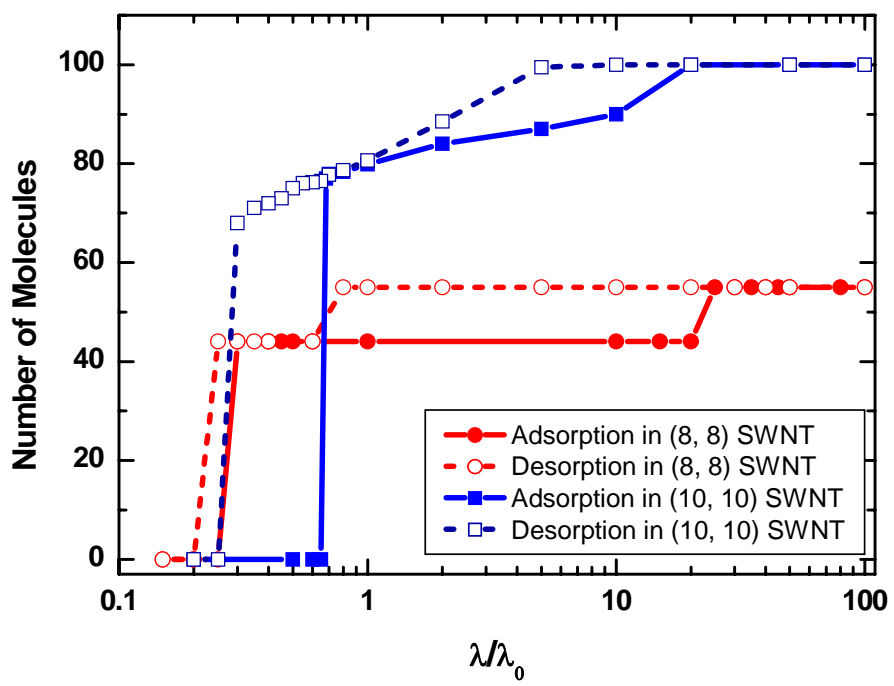

Fig. S1. Adsorption isotherms for water confined in $(8,8)$ and $(10,10)$ SWNT at $298 \mathrm{~K}$. $\lambda_{0}$ is the saturation activity at $298 \mathrm{~K}$ for SPC/E model. $\lambda / \lambda_{0}$ is the relative activity.

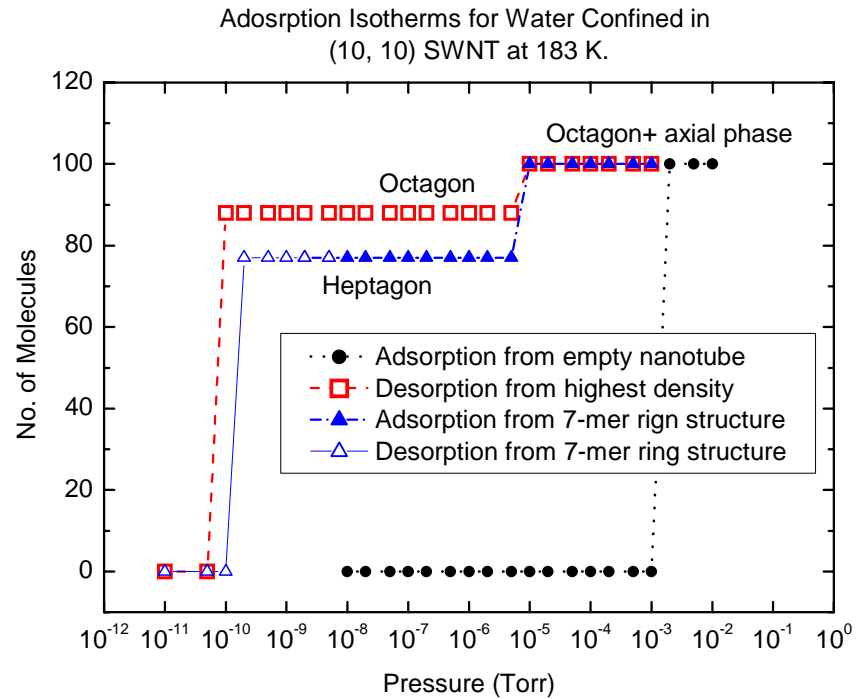

Fig. S2. Adsorption isotherms at $183 \mathrm{~K}$ for water confined in $(10,10) \mathrm{SWNT}$. 

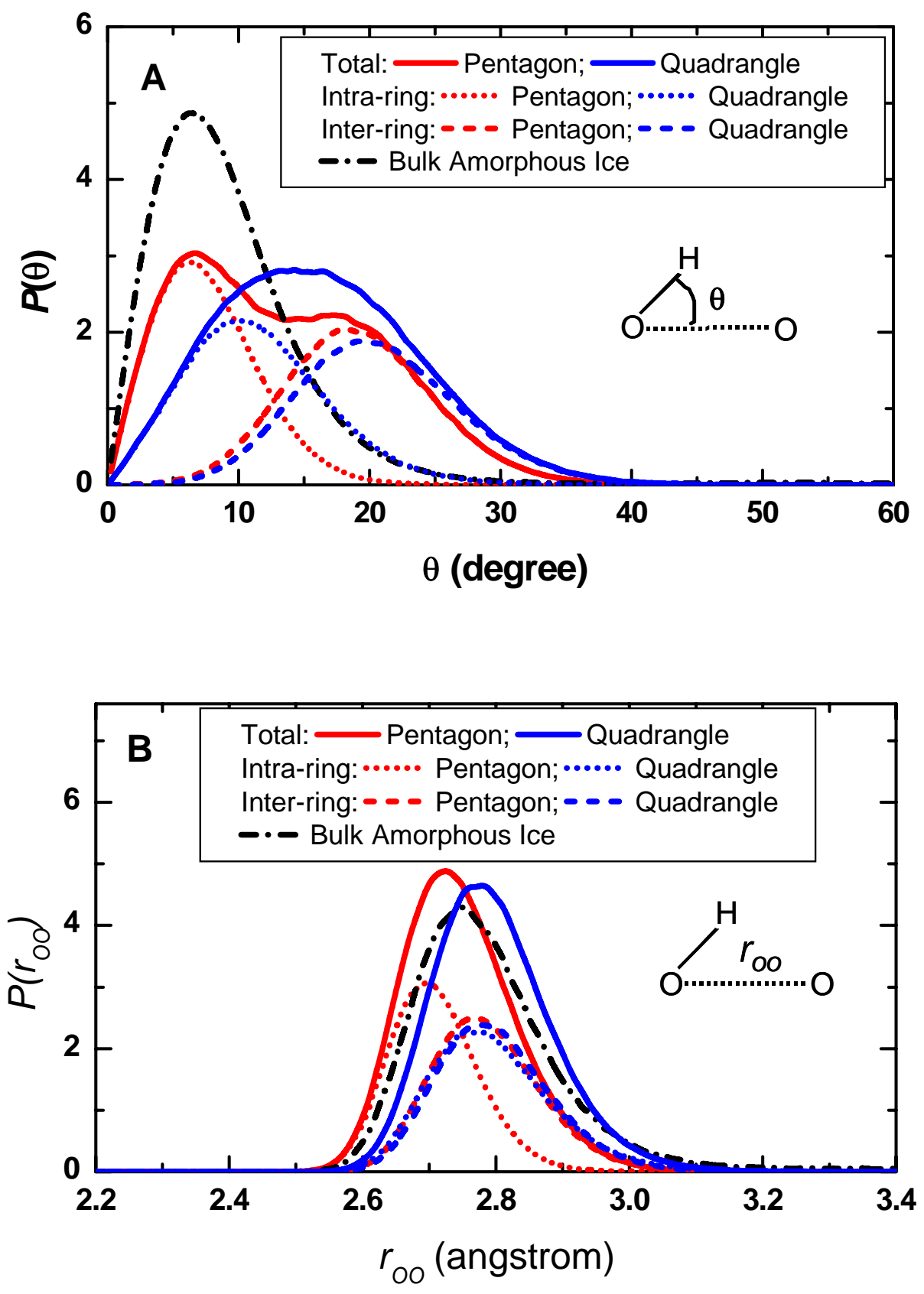

Fig. S3. Characteristics of hydrogen bonding in amorphous ice and $\mathrm{H}_{2} \mathrm{O}$ inside an $(8,8)$ SWNT computed from molecular simulations. (A) Hydrogen bond angle $(\mathrm{O}-\mathrm{OH})$ distribution computed from Monte Carlo simulation for bulk amorphous ice (dash-dot black line) and for $\mathrm{H}_{2} \mathrm{O}$ in an $(8,8)$ SWNT (solid lines). The intra-ring hydrogen bond angles are plotted as the dotted lines, the inter-ring hydrogen bond angles are represented by the dashed lines, and the solid line is the sum of the dotted and dashed lines of the same color. The inset shows the definition of the $\mathrm{O}-\mathrm{OH}$ angle $\theta$. (B) Oxygen-oxygen distance $(\mathrm{O}-\mathrm{OH})$ distribution computed from Monte Carlo simulation for bulk amorphous ice (dash-dot black line) and for $\mathrm{H}_{2} \mathrm{O}$ in an $(8,8)$ SWNT (solid lines). The intra-ring oxygen-oxygen distances are plotted as the dotted lines, the inter-ring oxygen-oxygen distances are represented by the dashed lines, and the solid line is the sum of the dotted and dashed lines of the same color. The inset shows the definition of the $\mathrm{O}-\mathrm{OH}$ distance $r_{\mathrm{OO}}$. 

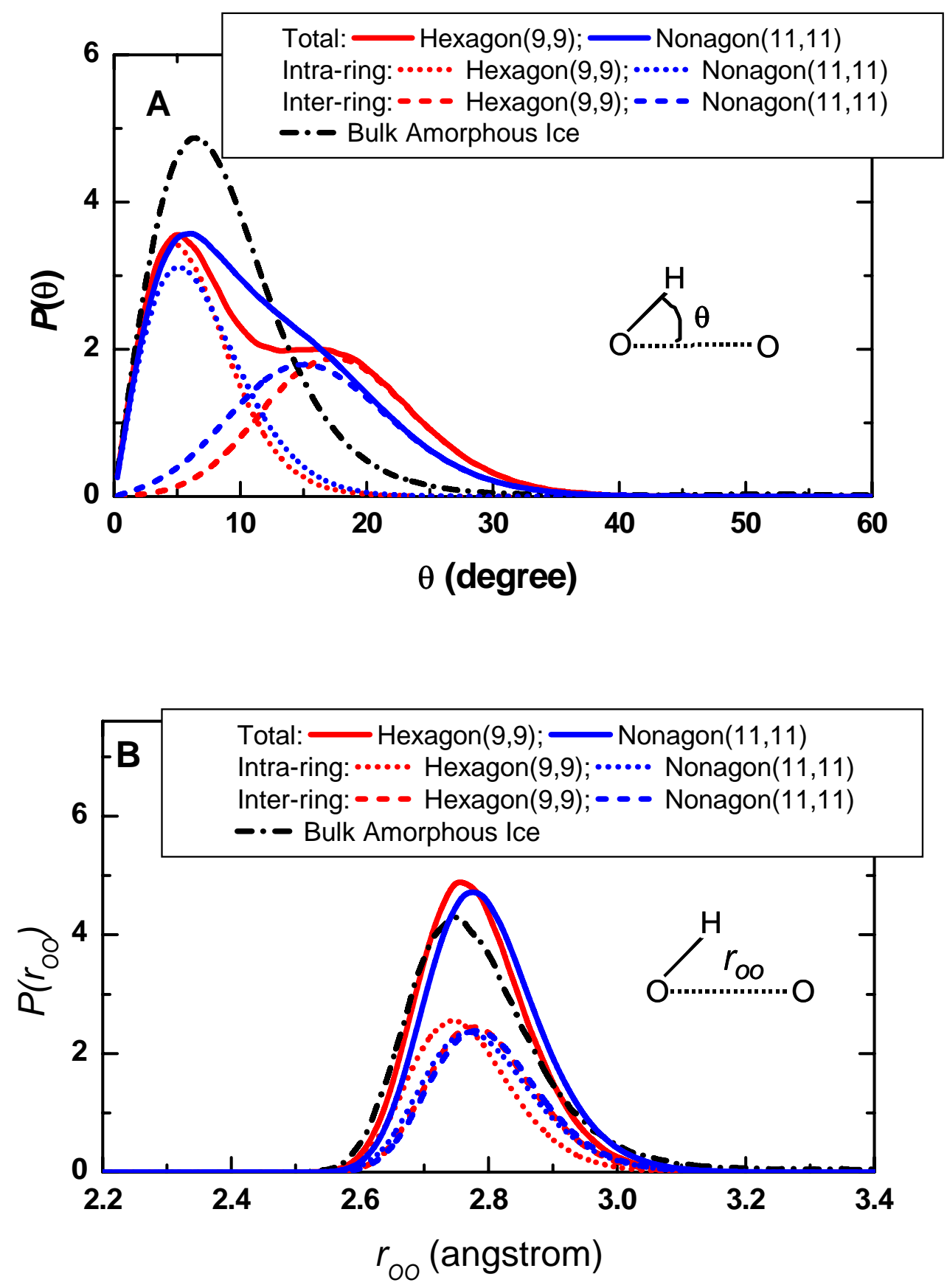

Fig. S4. Characteristics of hydrogen bonding in amorphous ice and $\mathrm{H}_{2} \mathrm{O}$ inside $(9,9)$ and $(11,11)$ SWNTs computed from molecular simulations. (A) Hydrogen bond angle $(\mathrm{O}-\mathrm{OH})$ distribution computed from Monte Carlo simulation for bulk amorphous ice (dash-dot black line) and for $\mathrm{H}_{2} \mathrm{O}$ in $(9,9)$ SWNT (red lines) and $(11,11)$ SWNT (blue lines). The intra-ring hydrogen bond angles are plotted as the dotted lines, the inter-ring hydrogen bond angles are represented by the dashed lines, and the solid line is the sum of the dotted and dashed lines of the same color. The inset shows the definition of the $\mathrm{O}-\mathrm{OH}$ angle $\theta$. (B) Oxygen-oxygen distance $(\mathrm{O}-\mathrm{OH})$ distribution computed from Monte Carlo simulation for bulk amorphous ice (dash-dot black line) and for $\mathrm{H}_{2} \mathrm{O}$ in $(9,9)$ SWNT (red lines) and $(11,11)$ SWNT (blue lines). The intraring oxygen-oxygen distances are plotted as the dotted lines, the inter-ring oxygen-oxygen distances are 
represented by the dashed lines, and the solid line is the sum of the dotted and dashed lines of the same color. The inset shows the definition of the $\mathrm{O}-\mathrm{OH}$ distance $r_{O O}$.

(2) Molecular dynamics simulations

We used the final configurations from the $\mathrm{MC}$ simulations as initial positions in the NVT MD calculations. Calculations were performed for bulk amorphous ice and water in nanotubes at $123 \mathrm{~K}$. The bulk ice simulation was equilibrated for $20 \mathrm{ps}$, followed by $100 \mathrm{ps}$ for data collection. Nanotube simulations were equilibrated for $400 \mathrm{ps}$ followed by $200 \mathrm{ps}$ for data taking. We used the Verlet leap-frog integrator with a 0.1 fs time step. This small integration time step is chosen to ensure correct accounting for the strong intra-molecular interactions. We used the Berendsen's thermostat ${ }^{8}$ with a coupling constant of $0.1 \mathrm{ps}$. The IR spectra were calculated from the velocity auto-correlation functions ${ }^{9}$. We used only the hydrogen atom velocity auto-correlation function to calculate the IR spectra for computational efficiency, as this has been shown to give good results ${ }^{9}$. The spectra were computed from Eq. (2) as described by Martí et al. ${ }^{9}$,

$$
I(\omega)=\pi^{-1} \omega^{-2} \int_{0}^{\infty}\langle\dot{M}(t) \cdot \dot{M}(0)\rangle \cos \omega t d t,
$$

where $\dot{M}(t)$ is the dipole moment velocity. The collective time correlation function of the dipole moment velocity is given by

$$
\langle\dot{M}(t) \cdot \dot{M}(0)\rangle=q^{2} Z_{\mathrm{HH}}^{c}(t)-4 q^{2} Z_{\mathrm{OH}}^{c}(t)+4 q^{2} Z_{\mathrm{OO}}^{c}(t),
$$

where $q$ is the charge on a hydrogen atom and $Z_{\mathrm{XY}}^{c}$ is the collective velocity correlation function between atoms $X$ and Y. Martí et al. ${ }^{9}$ have shown that Eq. (3) can be approximated by

$$
\langle\dot{M}(t) \cdot \dot{M}(0)\rangle \approx q^{2} Z_{\mathrm{HH}}^{c}(t)=\left\langle V_{\mathrm{H}}(t) \cdot V_{\mathrm{H}}(0)\right\rangle,
$$

where $V_{\mathrm{H}}$ is given by

$$
V_{\mathrm{H}}(t)=\sum_{i=1}^{2 N} v_{\mathrm{H}_{i}}(t)
$$

and $v_{\mathrm{H}_{i}}$ is the velocity of the $i$ th hydrogen atom and $\mathrm{N}$ is the total number of water molecules.

We have tested the effect of long-range electrostatic corrections on the calculated IR spectra by comparing our results using a simple truncation with spectra published by Martí et al. who used the Ewald method to account for long-range electrostatics ${ }^{9}$.We performed a simulation for bulk water at $298 \mathrm{~K}$ and density of $1 \mathrm{~g} / \mathrm{cm}^{3}$ using the SPC model, which was used by Martí et al. in their calculations ${ }^{9}$.The simulation cell is a cubic box with length equal to $2.86 \mathrm{~nm}$, containing 780 water molecules. We used Verlet leapfrog integrator with a 0.5 fs time step. The system was equilibrated for $200 \mathrm{ps}$ 
followed by $400 \mathrm{ps}$ for data collection, as these values were the same as those used by Martí et al. ${ }^{9}$. Our results are plotted in Fig. S5, along with data from Martí et al. ${ }^{9}$.

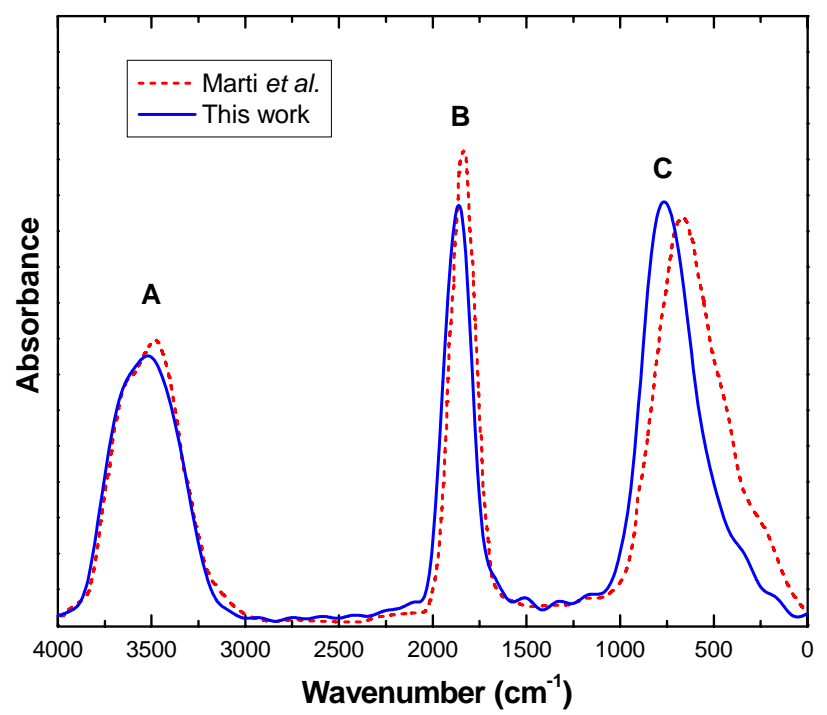

Fig. S5. The computed IR spectra for bulk water at ambient conditions. Solid blue line was computed without any long-range electrostatic corrections. The dashed red line was computed using the Ewald summation technique.

The two spectra plotted in Fig. S5 are very similar, especially for modes A and B. Mode $\mathrm{A}$ is due to the $\mathrm{O}-\mathrm{H}$ stretching motions and mode $\mathrm{B}$ is due to bond bending. These two modes are apparently not affected by long-range electrostatic corrections. Mode $\mathrm{C}$ from our calculations has a peak about $80 \mathrm{~cm}^{-1}$ higher in frequency than that computed by Martí et al. Mode C corresponds to librational motions, which are apparently more sensitive to the long-range electrostatic interactions. The excellent correspondence for modes A and B between calculations with no long-range electrostatic corrections and the published data using the Ewald technique verify that the O-H stretching modes are insensitive to long-range electrostatics for the large cutoff values we have used.

(3) Quantum mechanical calculations

We have used the Vienna Ab-initio Simulation Package (VASP) ${ }^{10-13}$ to compute DFT optimized structures and vibrational frequencies for gas phase water, water in ring structures, and water inside a SWNT. We have used the PBE generalized gradient exchange-correlation functional ${ }^{14}$ for all calculations. VASP is an implementation of periodic planewave DFT. We used projector augmented wave (PAW) pseudopotentials in our calculations with an energy cutoff $400 \mathrm{eV}$ and an augmentation charge cutoff of 645 $\mathrm{eV}$. Geometry optimizations were performed with the conjugate gradient algorithm as implemented within VASP. The energy convergence thresholds for the electronic structure self-consistent field calculations and the geometry optimizations were both set to $1 \times 10^{-4} \mathrm{eV}$. Vibrational frequencies were computed from numerical differentiation of the forces, with displacements set to $0.02 \AA$. The gas phase $\mathrm{H}_{2} \mathrm{O}$ antisymmetric $v_{3}$ and 
symmetric $v_{1}$ frequencies computed from VASP were $3830 \mathrm{~cm}^{-1}$ and $3702 \mathrm{~cm}^{-1}$, respectively. The gas-phase calculations were carried out by placing a single $\mathrm{H}_{2} \mathrm{O}$ molecule in a box $20 \AA$ on a side. Only the $\Gamma$ point was sampled in k-space. The 5 - and 7membered ring structures were placed in hexagonal supercells containing a single water ring. The 5-membered ring box size was $20 \AA \times 20 \AA \times 2.9 \AA$ and the 7-membered ring box size was $22 \AA \times 22 \AA \times 2.9 \AA$. A Monkhorst-Pack ${ }^{15}$ k-point grid of $1 \times 1 \times 6$ was used for both ring structures.

Calculations of a single $\mathrm{H}_{2} \mathrm{O}$ molecule inside a $(8,8)$ and a $(10,10)$ SWNT have been performed within VASP. Four SWNT unit cells were used for both calculations. The box sizes were $14.2 \AA \times 14.2 \AA \times 9.9 \AA$ and $16.8 \AA \times 16.8 \AA \times 9.9 \AA$ for the $(8,8)$ and $(10,10)$ SWNTs, respectively. The k-point grid was $1 \times 1 \times 3$. Stretching frequencies were $3814 \mathrm{~cm}^{-}$ ${ }^{1}$ and $3697 \mathrm{~cm}^{-1}$ for $\mathrm{H}_{2} \mathrm{O}$ in the $(8,8)$ SWNT and $3815 \mathrm{~cm}^{-1}$ and $3686 \mathrm{~cm}^{-1}$ for $\mathrm{H}_{2} \mathrm{O}$ in the $(10,10)$ SWNT.

Infrared intensities and vibrational frequencies for 5- and 7-membered water rings were computed with the PWscf package ${ }^{16}$. Ultrasoft pseudopotentials and the PBE functional were used. The hexagonal supercell dimensions for the PWscf calculations were virtually the same as for the VASP calculations and the k-point grid was $1 \times 1 \times 6$. The planewave energy cutoff for the PWscf calculations was $408 \mathrm{eV}$. Results from the PWscf calculations are plotted in Figures S6 and S7 for 5- and 7-membered rings, respectively.

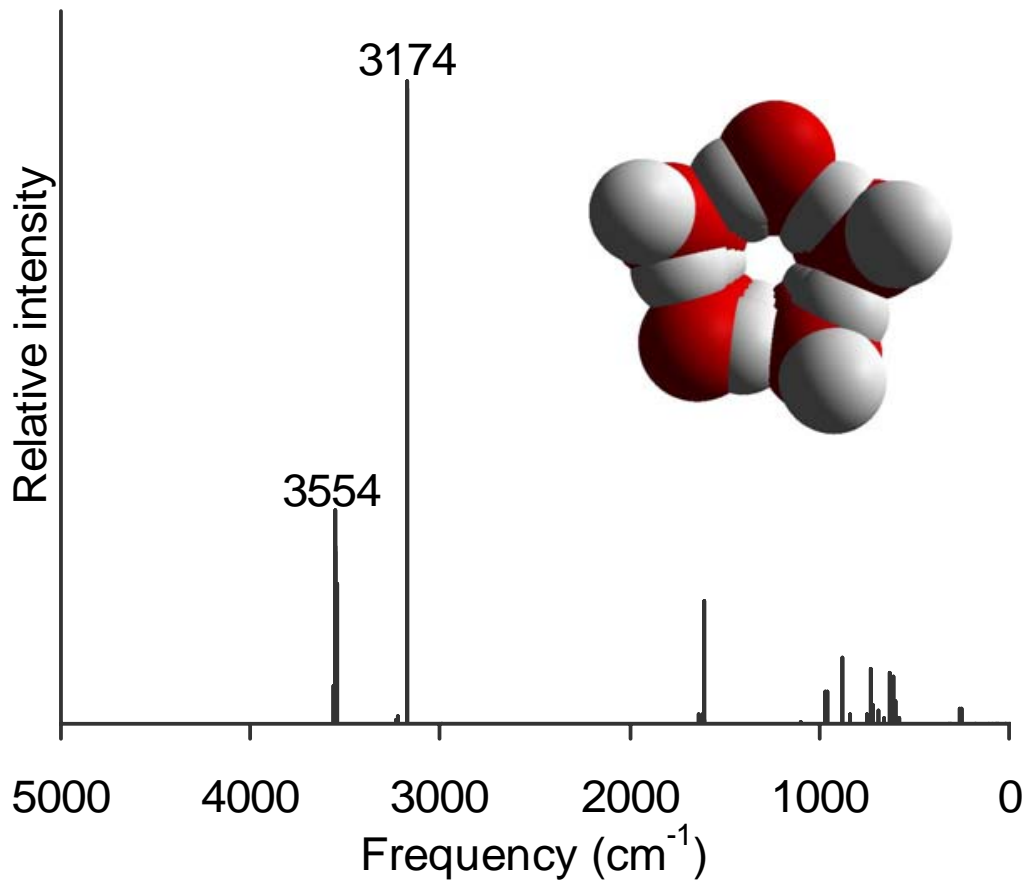

Fig. S6. The IR spectrum of the pentagonal stacked ring structure of water as computed from PWscf calculations using PBE-USPP. 


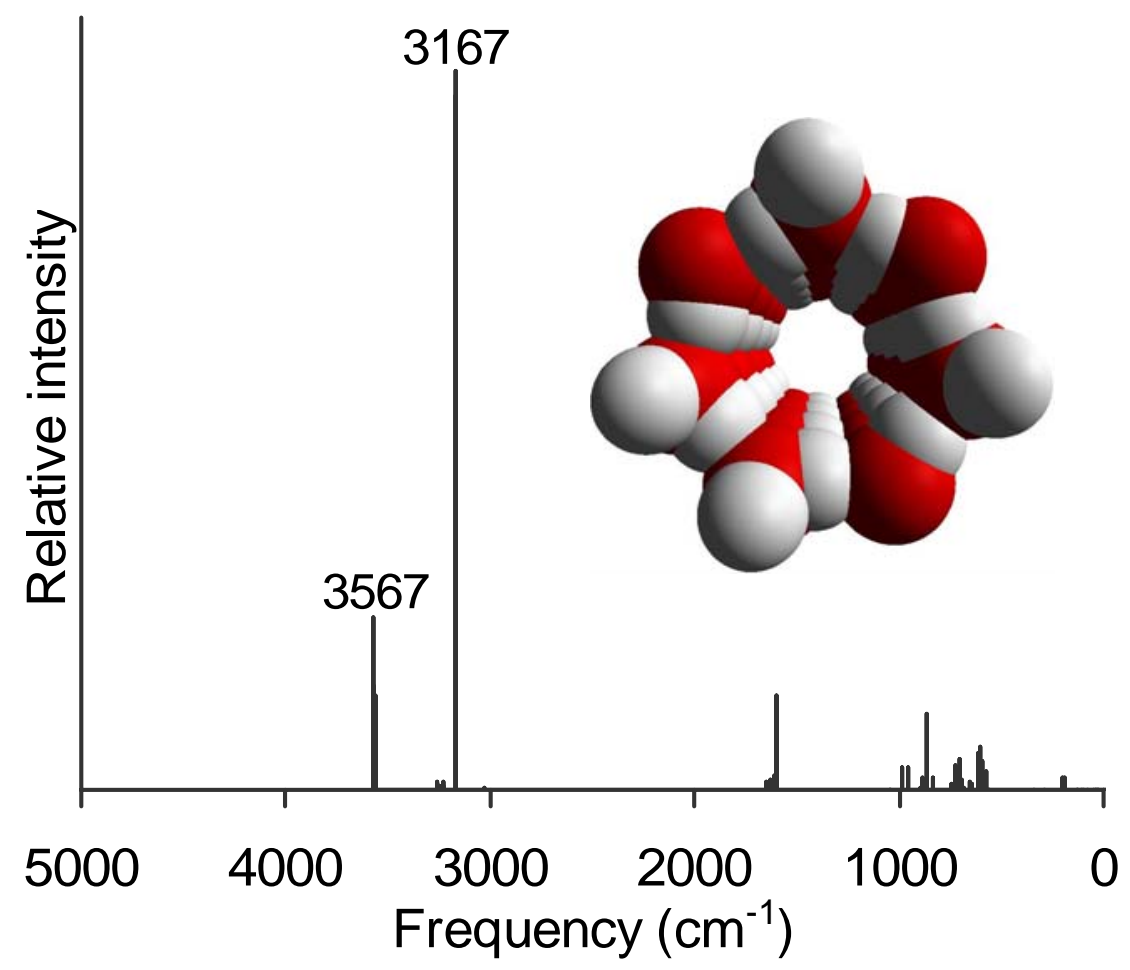

Fig. S7. The IR spectrum of the heptagonal stacked ring structure of water as computed from PWscf calculations using PBE-USPP.

The effect of the carbon nanotube on the vibrational intensities has been assessed by estimating the dynamic dipole moments for 5 -membred water rings inside a $(8,8)$ SWNT. Four unit cells of a $(8,8)$ SWNT were placed in a hexagonal supercell $(14.1 \AA \times 14.2 \AA \times$ $9.9 \AA$ ), and three $5-\mathrm{H}_{2} \mathrm{O}$ rings were located in the SWNT interior site. This arrangement gave a ring-ring spacing larger than the equilibrium structure observed in the $\mathrm{MC}$ and MD calculations; a much larger supercell would be required to obtain the correct ringring spacing. The relative IR intensity is proportional to the square of the dynamic dipole and the dynamic dipole can be estimated from the change in the Fermi energy resulting from displacements along the vibrational mode eigenvectors as discussed by Aizawa et al. ${ }^{17}$ and references therein. All calculations were performed within VASP. We found that the nanotube does not substantially change the IR intensities from those of the isolated water ring structures. This confirms that the nanotube is only a confining medium and that the experimentally observed $3507 \mathrm{~cm}^{-1}$ feature is a signature of geometric confinement, not of any specific interactions with the nanotube. 
Table S1. Vibrational frequencies of cylindrical $5 \mathrm{H}_{2} \mathrm{O}$ stacked rings.

\begin{tabular}{c|cc}
\hline & $\begin{array}{c}\text { VASP } \\
\text { PBE-PAW }\end{array}$ & $\begin{array}{c}P W s c f \\
P B E-U S P P\end{array}$ \\
\hline Supercell & Hexagonal & Hexagonal \\
& $(\mathrm{a}=\mathrm{b}=20.2 \AA, \mathrm{c}=2.9 \AA)$ & $(\mathrm{a}=\mathrm{b}=20.0 \AA, \mathrm{c}=2.9 \AA)$ \\
\hline Monkhorst-Pack & $1 \times 1 \times 6$ & $1 \times 1 \times 6$ \\
k-point grid & & \\
\hline Energy cutoff & $400 \mathrm{eV}$ & $408 \mathrm{eV}$ \\
\hline O-H stretching & 3564 & 3559 \\
frequenccy & 3561 & 3558 \\
& 3553 & 3555 \\
& 3552 & 3553 \\
& 3551 & 3547 \\
& 3234 & 3230 \\
& 3224 & 3226 \\
& 3145 & 3176 \\
& 3130 & 3171 \\
& 3012 & 2997 \\
\hline
\end{tabular}

Table S2. Vibrational frequencies of cylindrical $7 \mathrm{H}_{2} \mathrm{O}$ stacked rings.

\begin{tabular}{c|cc}
\hline & $\begin{array}{c}\text { VASP } \\
\text { SBE-PAW }\end{array}$ & $\begin{array}{c}P W s c f \\
P B E-U S P P\end{array}$ \\
\hline Monkhorst-Pack & Hexagonal & $\begin{array}{c}\text { Hexagonal } \\
(\mathrm{a}=\mathrm{b}=22.2 \AA, \mathrm{c}=2.9 \AA)\end{array}$ \\
k-point grid & $1 \times 1 \times 6$ & $\mathrm{a}=20.0 \AA, \mathrm{c}=2.9 \AA)$ \\
\hline Energy cutoff & & $1 \times 1 \times 6$ \\
\hline O-H stretching & $400 \mathrm{eV}$ & $408 \mathrm{eV}$ \\
frequenccy & 3584 & 3573 \\
& 3574 & 3570 \\
& 3571 & 3570 \\
& 3568 & 3568 \\
& 3566 & 3567 \\
& 3559 & 3567 \\
& 3558 & 3562 \\
& 3254 & 3255 \\
& 3250 & 3251 \\
& 3215 & 3233 \\
& 3208 & 3226 \\
& 3127 & 3170 \\
& 3109 & 3165 \\
& 3035 & 3028 \\
\hline
\end{tabular}

(4) Partially Filled Nanotubes 
The GCMC simulations indicate that it is unlikely that incomplete filling of the nanotubes will be observed. Nevertheless, we have explored the behavior of partially filled nanotubes by carrying out canonical (constant number of molecules) simulations. We find that the final structure at low temperatures is the same as the fully filled nanotube, with water forming a single stacked ring structure that partially fills the nanotube. This structure has free $\mathrm{OH}$ groups at the two ends of the stacked rings. If the nanotubes were not completely filled in experiments then a free $\mathrm{OH}$ mode should be observed. Because no free $\mathrm{OH}$ modes were observed in our experiments, we believe that it is very unlikely that the nanotubes are partially filled.

\section{Coordinates for Water in Nanotubes}

The coordinates for water in $(8,8)$ and $(10,10)$ SWNTs taken from simulation snapshots are given in files coord_8_8.xyz and coord_10_10.xyz, respectively. The coordinate units are given in Ångstroms.

\section{References}

(1) Berendsen, H. J. C.; Grigera, J. R.; Straatsma, T. P., The Missing Term in Effective Pair Potentials. J. Phys. Chem. 1987, 91, (24), 6269-6271.

(2) Praprotnik, M.; Janezic, D.; Mavri, J., Temperature Dependence of Water Vibrational Spectrum: A Molecular Dynamics Simulation Study. J. Phys. Chem. A 2004, 108, 1105611062.

(3) Toukan, K.; Rahman, A., Molecular-dynamics study of atomic motions in water. Phys. Rev. B 1985, 31, 2643-2648.

(4) Stan, G.; Cole, M. W., Low coverage adsorption in cylindrical pores. Surf. Sci. 1998, 395, (2-3), 280-291.

(5) Martí, J.; Gordillo, M. C., Temperature effects on the static and dynamic properties of liquid water inside nanotubes. Phys. Rev. E 2001, 64, (2), 021504.

(6) Allen, M. P.; Tildesley, D. J., Computer Simulation of Liquids. ed.; Oxford University Press: New York, 1987; 'Vol.' p.

(7) Frenkel, D.; Smit, B., Understanding Molecular Simulation: From Algorithms to Applications. ed.; Academic Press: San Diego, 2002; 'Vol.' p.

(8) Berendsen, H. J. C.; Postma, J. P. M.; Gunsteren, W. F. v.; DiNola, A.; Haak, J. R., Molecular dynamics with coupling to an external bath. J. Chem. Phys. 1984, 81, (8), 3684-3690.

(9) Martí, J.; Guàrdia, E.; Padró, J. A., Dielectric properties and infrared spectra of liquid water: Influence of the dynamic cross correlations. J. Chem. Phys. 1994, 101, (12), 10883-10891.

(10) Kresse, G.; Furthmueller, J., Efficient iterative schemes for ab initio total-energy calculations using a plane-wave basis set. Phys. Rev. B 1996, 54, 11169-11186.

(11) Kresse, G.; Furthmueller, J., VASP menu. Comput. Mat. Sci. 1996, 6, 15. 
(12) Kresse, G.; Hafner, J., Ab initio molecular dynamics for liquid metals. Phys. Rev. B 1993, 47, 558-561.

(13) Kresse, G.; Hafner, J., Ab initio molecular-dynamics simulation of the liquid-metalamorphous-semiconductor transition in germanium. Phys. Rev. B 1994, 49, 14251-14269. (14) Perdew, J. P.; Burke, K.; Ernzerhof, M., Generalized Gradient Approximation Made Simple. Phys. Rev. Lett. 1996, 77, 3865-3868.

(15) Monkhorst, H. J.; Pack, J. D., Special points for Brillouin-zone integrations. Phys. Rev. B 1976, 13, (12), 5188.

(16) Baroni, S.; Corso, A. D.; Gironcoli, S. d.; Giannozzi, P., http://www.pwscf.org. (17) Aizawa, H.; Morikawa, Y.; Tsuneyuki, S.; Fukutani, K.; Ohno, T., A densityfunctional study of the atomic structures and vibrational spectra of $\mathrm{NO} / \mathrm{Pt}\left(\begin{array}{lll}1 & 1 & 1\end{array}\right)$. Surf. Sci. 2002, 514, 394-403. 